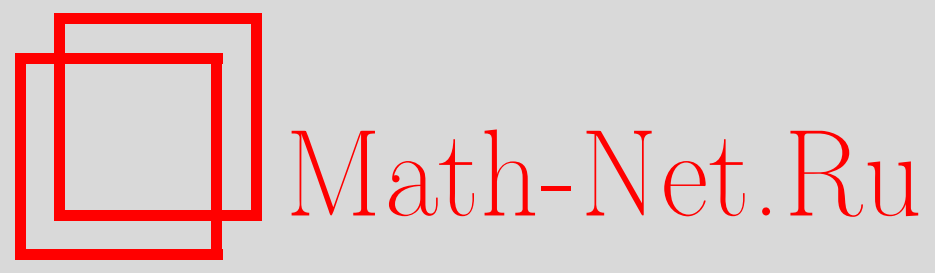

О. А. Козлитин, Полиномиальные преобразования GEOкольца простой характеристики, Дискрет. матем., 2004, том 16, выпуск 3, 105-117

DOI: https://doi.org/10.4213/dm166

Использование Общероссийского математического портала Math-Net.Ru подразумевает, что вы прочитали и согласны с пользовательским соглашением http://www . mathnet.ru/rus/agreement

Параметры загрузки:

IP : 54.198 .64 .247

26 апреля 2023 г., 05:53:03 


\title{
Полиномиальные преобразования GEO-кольца простой характеристики
}

\author{
(c) 2004 г. О. А. Козлитин
}

\begin{abstract}
Описаны полиномиальные и биективные полиномиальные преобразования конечного цепного не обязательно коммутативного кольца $S$, характеристика которого есть простое число. При дополнительном ограничении на индекс нильпотентности $e$ кольца $S$ найдено число (биективных) полиномиальных преобразований. Показано, что если кольцо $S$ некоммутативно, то среди полиномиальных преобразований $S$ нет транзитивных. Полученные результаты свидетельствуют о том, что при некоторых ограничениях на индекс нильпотентности $e$ число (биективных) полиномиальных преобразований некоммутативного кольца $S$ существенно больше числа описанных ранее (биективных) полиномиальных преобразований цепного коммутативного кольца той же мощности и характеристики.

Работа выполнена при поддержке гранта НШ 2358.2003.9 Президента Российской Федерации поддержки ведущих научных школ.
\end{abstract}

\section{1. Введение}

Пусть $S$ - конечное кольцо с единицей 1 . Для $a \in S$ обозначим через $\hat{a}$ преобразование кольца $S$, тождественно равное константе $a$. Если $R$ есть подкольцо $S$, то множество преобразований $\hat{a}$ по всем $a \in R$ обозначим через $\hat{R}$. В частности, $\hat{S}$ есть множество преобразований-констант кольца $S$.

Если $k=|S|$, то функщии, заданные на $S$ и принимающие значения в $S$, суть $k$-значные функции. Напомним понятие замыкания системы $k$-значных функций.

Рассмотрим так называемые функции-проекщии $e_{i}^{n}$,

$$
e_{i}^{n}\left(x_{1}, \ldots, x_{n}\right)=x_{i}, \quad i, n \in \mathbf{N}, \quad i \leqslant n .
$$

Множество всех проекций обозначим через $E$. Замыканием $\bar{G}$ системы $k$-значных функций $G$ называется минимальный по включению класс функций, удовлетворяющий следующим условиям.

(1) $G \cup E \subseteq \bar{G}$.

(2) Если $f\left(x_{1}, x_{2}, \ldots, x_{n}\right), g_{1}, g_{2}, \ldots, g_{n} \in \bar{G}$, то $f\left(g_{1}, g_{2}, \ldots, g_{n}\right) \in \bar{G}$.

(3) Если $f\left(x_{1}, x_{2}, \ldots, x_{n}\right) \in \bar{G}$, то все функции, полученные из $f$ добавлением или изъятием фиктивного переменного, также принадлежат $\bar{G}$. 
Полиномиальными называются преобразования кольца $S$, лежащие в замыкании системы $k$-значных функций $\hat{S} \cup\{t, \cdot\}$. Множество полиномиальных преобразований $S$ будем обозначать через $P(S)$. Биективные полиномиальные преобразования назовем также полиномиальными подстановками. Множество полиномиальных подстановок обозначим через $B(S)$.

Целью данной работы является описание множеств $P(S)$ и $B(S)$ в ситуации, когда $S$ есть кольцо Галуа-Эйзенштейна-Оре (конечное цепное кольцо, GEO-кольцо) простой характеристики $p$. Согласно [2] такое кольцо изоморфно $\mathbf{F}_{q}[z, \sigma] / z^{e}$, где $\mathbf{F}_{q}$ есть поле из $q=p^{t}$ элементов, $e$ и $t-$ натуральные числа, $\sigma$ - автоморфизм поля $\mathbf{F}_{q}, \mathbf{F}_{q}[z, \sigma]$ есть кольцо многочленов Оре, то есть кольцо многочленов над $\mathbf{F}_{q}$, где сложение обычное, а умножение определяется равенством

$$
z \alpha=\sigma(\alpha) z
$$

для всех $\alpha \in \mathbf{F}_{q}$.

В коммутативном случае указанная задача решена в [4]. Чтобы обобщить эти результаты на некоммутативный случай, охарактеризуем множество $P(S)$ в терминах теории колец.

Пусть $\Sigma$ - множество всех преобразований кольца $S$. Наделим $\Sigma$ структурой кольца: введем на $\Sigma$ операции сложения и умножения. Для любых $f_{1}, f_{2} \in \Sigma, a \in S$ положим

$$
\left(f_{1}+f_{2}\right)(a)=f_{1}(a)+f_{2}(a), \quad\left(f_{1} \cdot f_{2}\right)(a)=f_{1}(a) \cdot f_{2}(a) .
$$

Очевидно, что $(\Sigma,+, \cdot)$ - конечное кольцо с единицей $\hat{1}$.

Для всякого $\Gamma \subseteq \Sigma$ подкольцо кольца $(\Sigma,+, \cdot)$, порожденное $\Gamma$, обозначим [ $\Gamma]$. Тождественное преобразование кольца $S$ обозначим $\varepsilon$. Тогда $P(S)$ как множество совпадает с подкольцом $[\hat{S} \cup\{\varepsilon\}]$ кольца $\Sigma$. В дальнейшем под кольцом $P(S)$ будем понимать кольцо $[\hat{S} \cup\{\varepsilon\}]$.

\section{2. Предварительный результат о полиномиа́льности}

Выберем и зафиксируем некоторый изоморфизм колец $\mathbf{F}_{q}[z, \sigma] / z^{e}$ и $S$. Образ элемента $z$ при этом изоморфизме обозначим через $\pi$. Очевидно, что имеет место однозначное разложение любого элемента $a \in S$

$$
a=\sum_{i=0}^{e-1} a_{i} \pi^{i}
$$

где $a_{i} \in \mathbf{F}_{q}$ для всех $i=0,1, \ldots, e-1$.

Пусть $\delta$ есть порядок автоморфизма $\sigma$ в группе автоморфизмов поля $\mathbf{F}_{q}$. Для всех $i=0,1, \ldots, e-1$ обозначим через $\varphi_{i}$ преобразование кольца $S$, переводящее всякий элемент $a \in S$ в коэффициент $a_{i}$ из разложения (1) элемента $a$. Так как $S$ есть левое векторное пространство над полем $\mathbf{F}_{q}$, можно считать, что $\varphi_{i} \in \Sigma$. Для всех $j=0,1, \ldots, \delta$ определим преобразование $\varepsilon_{j} \in \Sigma$ равенством

$$
\varepsilon_{j}=\sum_{i \geqslant 0} \varphi_{j+i \delta} \hat{\pi}^{j+i \delta}
$$

Существует единственное число $r \in\{1, \ldots, t\}$ такое, что для любого $u \in \mathbf{F}_{q}$ выполняется равенство $\sigma(u)=u^{p^{r}}$. Для всех $i \in\{1, \ldots, \delta-1\}$ обозначим через $k_{i}$ остаток от 
деления $r i$ на $t$. Для краткости будем обозначать величину $p^{k_{i}}$ через $d_{i}, i=1, \ldots, \delta-1$. Тогда для любых $u \in \mathbf{F}_{q}, i \in\{1, \ldots, \delta-1\}$ справедливо равенство $\sigma^{i}(u)=u^{d_{i}}$. Очевидно, что для всех $i \in\{1, \ldots, \delta-1\}$ выполняется неравенство $1<d_{i}<p^{t}$, и разным $i \in\{1, \ldots, \delta-1\}$ соответствуют разные $d_{i}$. Обозначим через $M_{0}$ множество преобразований кольца $S$ вида

$$
\sum_{i: \operatorname{Res}_{\delta}(i) \neq 0} \alpha_{\operatorname{Res}_{\delta}(i)} \varphi_{i} \hat{\pi}^{i},
$$

где $\alpha_{j} \in \hat{\mathbf{F}}_{q}, \operatorname{Res}_{\delta}(i)$ есть остаток от деления $i$ на $\delta$. Очевидно, что $M_{0}$ есть левое векторное пространство над полем $\hat{\mathbf{F}}_{q}$, изоморфное $\mathbf{F}_{q} \mathbf{F}_{q}^{\delta-1}$, где $\mathbf{F}_{q}^{\delta-1}$ есть $(\delta-1)$-я декартова степень $\mathbf{F}_{q}$.

Полиномиальные преобразования $\alpha \varepsilon-\varepsilon \alpha, \alpha \in \hat{\mathbf{F}}_{q}$, имеют вид

$$
\sum_{i: \operatorname{Res}_{\delta}(i) \neq 0}\left(\alpha-\sigma^{\operatorname{Res} \delta(i)}(\alpha)\right) \varphi_{i} \hat{\pi}^{i} .
$$

Отсюда следует, что $\alpha \varepsilon-\varepsilon \alpha \in M_{0}$ для всех $\alpha \in \hat{\mathbf{F}}_{q}$. Размерность $M_{0}$ равна $\delta-1$, поэтому, если в $M_{0}$ есть линейно независимая система из $\delta-1$ векторов вида $\alpha \varepsilon-\varepsilon \alpha$, то в $M_{0}$ есть полиномиальный базис и все преобразования из $M_{0}$ полиномиальны. Иными словами, для включения $M_{0} \subseteq P(S)$ достаточно существование таких $\alpha_{1}, \alpha_{2}, \ldots, \alpha_{\delta-1} \in \mathbf{F}_{q}$, что

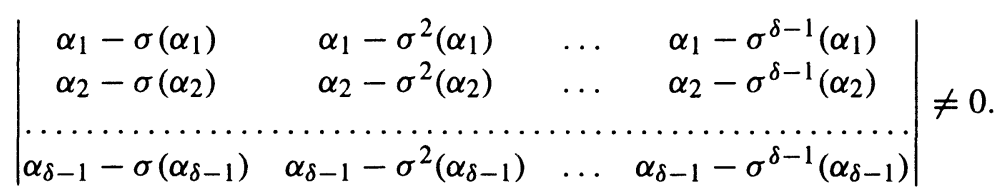

Существование $\alpha_{1}, \alpha_{2}, \ldots, \alpha_{\delta-1}$ с указанным свойством вытекает из следующей леммы.

Лемма 1. Для любого $s \in\{1, \ldots, \delta-1\}$ существуют элементы $\alpha_{1}, \alpha_{2}, \ldots, \alpha_{s}$ поля $\mathbf{F}_{q}$ такие, что

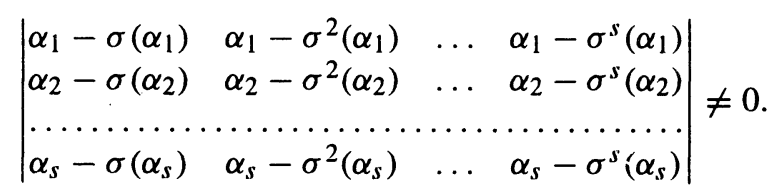

Доказательство. Докажем справедливость леммы индукцией по $s$.

В случае $s=1$ утверждение очевидно: существует $\alpha_{1}$ со свойством $\alpha_{1} \neq \sigma\left(\alpha_{1}\right)$. Пусть теперь $1 \leqslant s \leqslant \delta-2$ и существуют элементы $\alpha_{1}, \alpha_{2}, \ldots, \alpha_{s}$ с указанным в условии свойством. Рассмотрим многочлен

$$
\left|\begin{array}{cccc}
\alpha_{1}-\sigma\left(\alpha_{1}\right) & \alpha_{1}-\sigma^{2}\left(\alpha_{1}\right) & \ldots & \alpha_{1}-\sigma^{s+1}\left(\alpha_{1}\right) \\
\ldots \ldots \ldots \ldots \ldots \ldots \ldots \ldots \ldots \ldots \ldots \ldots \ldots \ldots \ldots \ldots \ldots \ldots \ldots \ldots \ldots \ldots & \ldots \ldots \ldots \\
\alpha_{s}-\sigma\left(\alpha_{s}\right) & \alpha_{s}-\sigma^{2}\left(\alpha_{s}\right) & \ldots & \alpha_{s}-\sigma^{s+1}\left(\alpha_{s}\right) \\
x-x^{d_{1}} & x-x^{d_{2}} & \ldots & x-x^{d_{s+1}}
\end{array}\right|
$$

Этот многочлен ненулевой, так как коэффициент при $x^{d_{s+1}}$ равен с точностью до знака минору, стоящему в первых $s$ строках и $s$ столбцах, а данный минор отличен от нуля по предположению индукции. Степень многочлена (2) строго меньше $p^{t}=q$, а значит, существует элемент $\alpha_{s+1} \in \mathbf{F}_{q}$, не являющийся его корнем. Лемма доказана. 
Таким образом, $M_{0} \subseteq P(S)$, а поскольку $\varepsilon_{j} \in M_{0}$ для всех $j \in\{1, \ldots, \delta-1\}$ и выполняется равенство

$$
\varepsilon_{0}=\varepsilon-\sum_{j=1}^{\delta-1} \varepsilon_{j}
$$

справедливо следующее утверждение.

Следствие 1. Преобразования $\varepsilon_{0}, \varepsilon_{1}, \ldots, \varepsilon_{\delta-1}$ полиномиальны.

В следующем параграфе доказанный факт будет использован для описания полиномиальных преобразований кольца $S$.

\section{3. Модуль полиномиальных преобразований.}

Пусть $\mathfrak{R}$ - подкольцо $\Sigma$ и $\Gamma \subseteq \Sigma$. Через $\mathfrak{R}[\Gamma]$ обозначим множество элементов $f\left(\gamma_{1}, \gamma_{2}, \ldots, \gamma_{n}\right)$ по всем натуральным $n$, многочленам $f \in \mathfrak{R}\left[x_{1}, x_{2}, \ldots, x_{n}\right]$ и элементам $\gamma_{i} \in \Gamma, i=1, \ldots, n$. Согласно [1] подполугруппу полугруппы $(\Sigma, \cdot)$, порожденную $\Gamma$, будем обозначать через $[\Gamma\rangle$.

Обозначим через $K(\hat{S}, \varepsilon)$ множество преобразований $\alpha \varepsilon-\varepsilon \alpha$ по всем $\alpha \in \hat{S}$, а через $H$ полугруппу $[\hat{1}, \varepsilon, K(\hat{S}, \varepsilon)\rangle$. Легко видеть, что

$$
\hat{S}[\varepsilon, K(\hat{S}, \varepsilon)]
$$

есть множество конечных сумм вида $\sum_{i} \alpha_{i} \omega_{i}$, где $\alpha_{i} \in \hat{S}, \omega_{i} \in H$. Нам понадобится следующая лемма.

Лемма 2. Справедливо равенство $[\hat{S} \cup\{\varepsilon\}]=\hat{S}[\varepsilon, K(\hat{S}, \varepsilon)]$.

Доказательство. Очевидно, что $[\hat{S} \cup\{\varepsilon\}] \supseteq \hat{S}[\varepsilon, K(\hat{S}, \varepsilon)]$ и $\hat{S}[\varepsilon, K(\hat{S}, \varepsilon)]$ есть абелева группа по сложению. Для того чтобы доказать замкнутость $\hat{S}[\varepsilon, K(\hat{S}, \varepsilon)]$ по умножению, достаточно показать, что для любых $\alpha, \beta \in \hat{S}$ и $\omega^{\prime}, \omega^{\prime \prime} \in H$ выполняется включение $\alpha \omega^{\prime} \beta \omega^{\prime \prime} \in \hat{S}[\varepsilon, K(\hat{S}, \varepsilon)]$. Для этого, в свою очередь, достаточно, чтобы $\omega \alpha \in \hat{S}[\varepsilon, K(\hat{S}, \varepsilon)]$ для всех $\alpha \in \hat{S}, \omega \in H$. Иными словами, будем доказывать, что $\gamma_{1} \gamma_{2} \ldots \gamma_{s} \alpha \in \hat{S}[\varepsilon, K(\hat{S}, \varepsilon)]$ для любых $s \geqslant 1, \gamma_{i} \in K(\hat{S}, \varepsilon) \cup\{\hat{1}, \varepsilon\}, \alpha \in \hat{S}$.

Доказательство проведем индукщией по параметру $s$. Пусть $s=1$. Если $\gamma_{1}=\varepsilon$, то

$$
\gamma_{1} \alpha=\varepsilon \alpha=\alpha \varepsilon-(\alpha \varepsilon-\varepsilon \alpha) \in \hat{S}[\varepsilon, K(\hat{S}, \varepsilon)] .
$$

Если $\gamma_{1} \in K(\hat{S}, \varepsilon)$, то для некоторого $\beta \in \hat{S}$

$$
\gamma_{1} \alpha=(\beta \varepsilon-\varepsilon \beta) \alpha=\beta(\varepsilon \alpha)-\varepsilon(\beta \alpha) \in \hat{S}[\varepsilon, K(\hat{S}, \varepsilon)],
$$

так как, согласно доказанному, $\varepsilon \alpha \in \hat{S}[\varepsilon, K(\hat{S}, \varepsilon)]$ и $\varepsilon(\beta \alpha) \in \hat{S}[\varepsilon, K(\hat{S}, \varepsilon)]$. В случае $\gamma_{1}=\hat{1}$ доказываемое утверждение очевидно.

Пусть теперь утверждение верно для значения $s=s_{0}$, докажем его для $s=s_{0}+1$. Справедливо равенство

$$
\gamma_{1} \gamma_{2} \ldots \gamma_{s_{0}}\left(\gamma_{s_{0}+1} \alpha\right)=\gamma_{1} \gamma_{2} \ldots \gamma_{s_{0}} \sum_{i} \alpha_{i} \omega_{i}=\sum_{i}\left(\gamma_{1} \gamma_{2} \ldots \gamma_{s_{0}} \alpha_{i}\right) \omega_{i},
$$


где $\alpha_{i} \in \hat{S}, \omega_{i} \in H$. По предположению индукции

$$
\gamma_{1} \gamma_{2} \ldots \gamma_{s_{0}} \alpha_{i}=\sum_{j} \alpha_{i, j} \omega_{i, j}
$$

для некоторых $\alpha_{i, j} \in \hat{S}, \omega_{i, j} \in H$. Тогда

$$
\gamma_{1} \gamma_{2} \ldots \gamma_{s_{0}} \gamma_{s_{0}+1} \alpha=\sum_{i, j} \alpha_{i, j} \omega_{i, j} \omega_{i} \in \hat{S}[\varepsilon, K(\hat{S}, \varepsilon)] .
$$

Таким образом, $\hat{S}[\varepsilon, K(\hat{S}, \varepsilon)]$ есть подкольцо $\Sigma$, включающее $\hat{S} \cup\{\varepsilon\}$. Следовательно, $[\hat{S} \cup\{\varepsilon\}] \subseteq \hat{S}[\varepsilon, K(\hat{S}, \varepsilon)]$.

Лемма доказана.

Описание полиномиальных преобразований GEO-кольца простой характеристики дает следующая тесрема.

Теорема 1. Если $S$ есть GEO-кольчо простой характеристики, то кольца

$$
M_{1}=\hat{S}[\varepsilon, K(\hat{S}, \varepsilon)], \quad M_{2}=\hat{S}\left[\hat{\pi}, \varepsilon_{0}, \varepsilon_{1}, \ldots, \varepsilon_{\delta-1}\right], \quad M_{3}=\hat{\mathbf{F}}_{q}\left[\hat{\pi}, \varphi_{0}, \varepsilon_{1}, \ldots, \varepsilon_{\delta}\right]
$$

равны между собой и совпадают $с(P(S),+, \cdot)$.

Доказательство. В силу леммы 2 достаточно показать, что имеет место цепочка включений

$$
M_{1} \supseteq M_{2} \supseteq M_{3} \supseteq M_{1} \text {. }
$$

Включение $M_{1} \supseteq M_{2}$ справедливо в силу следствия 1 .

Для доказательства включения $M_{2} \supseteq M_{3}$ достаточно показать полиномиальность преобразования $\varphi_{0}$ (поскольку $\varepsilon_{0}=\varphi_{0}+\varepsilon_{\delta}$, из полиномиальности $\varphi_{0}$ следует полиномиальность $\varepsilon_{\delta}$ ). Поскольку элементы вида $a \pi^{i \delta}, a \in \mathbf{F}_{q}, i \in \mathbf{N}_{0}$, перестановочны между собой, для всякого натурального $n$

$$
\varepsilon_{0}(a)^{q^{n}}=\left(\sum_{i \geqslant 0} a_{i \delta} \pi^{i \delta}\right)^{q^{n}}=\sum_{i \geqslant 0} a_{i \delta} \pi^{i \delta q^{n}}
$$

где $a_{j} \in \mathbf{F}_{q}-$ коэффициенты из разложения (1) элемента $a \in S$. Если $n$ таково, что $\delta q^{n} \geqslant e$, то $\varepsilon_{0}(a)^{q^{n}}=a_{0}=\varphi_{0}(a)$ для всех $a \in S$, то есть преобразование $\varphi_{0}=\varepsilon_{0}^{q^{n}}$ полиномиально.

Для доказательства включения $M_{3} \supseteq M_{1}$ достаточно показать, что для всех $a \in \mathbf{F}_{q}$ и $i \in \mathbf{N}_{0}$ преобразования $\hat{a} \hat{\pi}^{i} \varepsilon$ и $\varepsilon \hat{a} \hat{\pi}^{i}$ принадлежат $M_{3}$. Так как

$$
\varepsilon=\varphi_{0}+\sum_{j=1}^{\delta} \varepsilon_{j}
$$

справедливо включение $\hat{a} \hat{\pi}^{i} \varepsilon \in M_{3}$. Преобразование $\varepsilon \hat{a} \hat{\pi}^{i}$ можно представить в виде

$$
\hat{a} \varphi_{0} \hat{\pi}^{i}+\sum_{j=1}^{\delta} \widehat{\sigma^{j}(a)} \varepsilon_{j} \hat{\pi}^{i}
$$

и значит, оно принадлежит $M_{3}$.

Теорема доказана. 
Поскольку $\hat{\pi} \varphi_{0}=\sigma\left(\varphi_{0}\right) \hat{\pi}$ и для всех $j \in\{1, \ldots, \delta\}$

$$
\varepsilon_{j} \varphi_{0}=\sigma^{j}\left(\varphi_{0}\right) \varepsilon_{j}
$$

справедливо равенство

$$
P(S)=\hat{\mathbf{F}}_{q}\left[\hat{\pi}, \varphi_{0}, \varepsilon_{1}, \ldots, \varepsilon_{\delta}\right]=\hat{\mathbf{F}}_{q}\left[\varphi_{0}\right]\left[\hat{\pi}, \varepsilon_{1}, \ldots, \varepsilon_{\delta}\right]
$$

Для любого $f$-преобразования $\mathbf{F}_{q}$ и $g \in P(S)$ определим произведение $f g$ равенством

$$
f g(a)=f\left(\varphi_{0}(a)\right) g(a)
$$

для любого $a \in S$. Из равенства $P(S)=\hat{\mathbf{F}}_{q}\left[\varphi_{0}\right]\left[\hat{\pi}, \varepsilon_{1}, \ldots, \varepsilon_{\delta}\right]$ следует, что $f g \in P(S)$. Если $P\left(\mathbf{F}_{q}\right)$ - кольцо преобразований $\mathbf{F}_{q}$ и $T$ есть система всех различных ненулевых элементов полугруппы $\left[\hat{1}, \hat{\pi}, \varepsilon_{1}, \ldots, \varepsilon_{\delta}\right\rangle$, то $P(S)$ есть левый $P\left(\mathbf{F}_{q}\right)$-модуль с системой образующих $T$.

Для дальнейшего изложения понадобятся некоторые понятия теории модулей. Пусть $R$ - конечное коммутативное кольцо с единицей, $R$ M - конечный левый $R$-модуль и $\Gamma$ некоторая система векторов модуля ${ }_{R} M$. Система $\Gamma$ называется свободной (над $R$ ), если между ее элементами не существует нетривиального линейного соотношения над $R$, и несократимой (над $R$ ), если ни один из ее элементов не является линейной комбинацией остальных. Если модуль $R_{R} M$ имеет свободную систему образующих, то он называется свободным, а сама эта система называется базисом модуля ${ }_{R} M$. Мощность базиса свободного модуля называется рангом этого модуля (для ранга используется обозначение rank $\left._{R} M\right)$. Справедлива следующая лемма.

Лемма 3. Любая подсистема $Г$ системы $T$ свободна тогда и только тогда, когда она несократима.

Доказательство. То, что всякая сократимая система несвободна, очевидно. Наоборот, пусть система $Г$ несвободна:

$$
\sum_{i=1}^{n} a_{i} \gamma_{i}=0
$$

где $a_{i} \in P\left(\mathbf{F}_{q}\right) \backslash\{0\}, \gamma_{i} \in \Gamma, n \geqslant 1$. Выберем элемент $u \in S$ такой, что не все $a_{i}$ в точке $\varphi_{0}(u)=u_{0}$ равны нулю. Не ограничивая общности, можно считать, что существует число $k \in\{1, \ldots, n\}$ такое, что $a_{i}\left(u_{0}\right) \neq 0$ для всех $i \in\{1, \ldots, k\}$, и

$$
\sum_{i=1}^{k} a_{i}\left(u_{0}\right) \gamma_{i}(u)=0 .
$$

Таким образом,

$$
\gamma_{1}(u)=-\sum_{i=2}^{k} a_{1}\left(u_{0}\right)^{-1} a_{i}\left(u_{0}\right) \gamma_{i}(u)
$$

и, более того,

$$
\gamma_{1}(v)=-\sum_{i=2}^{k} a_{1}\left(u_{0}\right)^{-1} a_{i}\left(u_{0}\right) \gamma_{i}(v)
$$


для всех $v$ таких, что $\varphi_{0}(v)=u_{0}$. Однако, $\gamma_{i} \in T$, и следовательно, $\gamma_{i}(x)$ не зависит от $\varphi_{0}(x)=x_{0}$. Поэтому, если $c_{i}=-a_{1}\left(u_{0}\right)^{-1} a_{i}\left(u_{0}\right) \in \mathbf{F}_{q}$ для всех $i \in\{2, \ldots, k\}$, то

$$
\gamma_{1}=\sum_{i=2}^{k} \hat{c}_{i} \gamma_{i}
$$

Таким образом, если система Г несвободна, то она сократима.

Лемма доказана.

Чтобы сформулировать и доказать основной результат этого параграфа, понадобятся еще несколько определений. Всякому преобразованию $f \in \Sigma$ сопоставим систему координатных функций $f_{s}$ над полем $\mathbf{F}_{q}$,

$$
f_{s}=\varphi_{s}(f), \quad s=0,1, \ldots, e-1 .
$$

Нормой преобразования $f \neq 0$ (для нее используем обозначение $\|f\|$ ) назовем минимальное $s$ такое, что $f_{s} \neq 0$. Приведенным назовем многочлен над полем $\mathbf{F}_{q}$, имеющий по каждому переменному степень не выше $q-1$. Хорошо известно [3], что всякая функция над $\mathbf{F}_{q}$ задается единственным приведенным многочленом.

Теорема 2. Пусть $S$ есть GEO-кольчо простой характеристики. Тогда $P(S)$ есть свободный левый $P\left(\mathbf{F}_{q}\right)$-модуль, причем $T$ является его системой образующих и содержит базис. Если дополнительно известно, что $e \leqslant 1+\delta$, то $T$ является базисом модуля $P\left(\mathbf{F}_{q}\right) P(S)$.

Доказательство. Согласно лемме 3 максимальная несократимая подсистема $T$ есть базис $P\left(\mathbf{F}_{q}\right) P(S)$, а значит, модуль $P\left(\mathbf{F}_{q}\right) P(S)$ свободный.

Пусть теперь $e \leqslant 1+\delta$. Доказывать утверждение теоремы будем от противного. Предположим, что система $T=\left\{\gamma_{1}, \gamma_{2}, \ldots, \gamma_{n}\right\}$ несвободна. Перенумеровав элементы $T$, получим, что существуют $k \in\{1, \ldots, n\}$ и $a_{1}, a_{2}, \ldots, a_{k} \in P\left(\mathbf{F}_{q}\right) \backslash\{0\}$ такие, что

$$
\sum_{i=1}^{k} a_{i} \gamma_{i}=0
$$

Пусть

$$
s_{0}=\min \left\{\left\|\gamma_{1}\right\|,\left\|\gamma_{2}\right\|, \ldots,\left\|\gamma_{k}\right\|\right\}
$$

Не ограничивая общности, будем считать, что существует число $k_{0} \in\{1, \ldots, k\}$ такое, что

$$
\left\|\gamma_{1}\right\|=\left\|\gamma_{2}\right\|=\ldots=\left\|\gamma_{k_{0}}\right\|=s_{0}
$$

и $\left\|\gamma_{i}\right\|>s_{0}$ для всех $i>k_{0}$. Поскольку $e \leqslant 1+\delta$, справедливо неравенство $s_{0} \leqslant \delta$. Рассмотрим $s_{0}$-ю координатную функцию преобразования $f=\sum_{i=1}^{k} a_{i} \gamma_{i}$. Так как $e \leqslant 1+\delta$, для всех $i \in\left\{1, \ldots, k_{0}\right\}$ справедливо равенство $\gamma_{i}=\omega_{i}\left(\varphi_{1}, \varphi_{2}, \ldots, \varphi_{s_{0}}\right) \hat{\pi}^{s_{0}}$, где $\omega_{i}$ - некоторая ненулевая функция, заданная на $\mathbf{F}_{q}$ и принимающая значения в $\mathbf{F}_{q}$ (в случае $s_{0}=0$ считаем, что $\omega_{i}=1$ ). Таким образом, справедливо равенство функций

$$
f_{s_{0}}=\sum_{i=1}^{k_{0}} a_{i}\left(\varphi_{0}\right) \omega_{i}\left(\varphi_{1}, \varphi_{2}, \ldots, \varphi_{s_{0}}\right)=0 .
$$


Можно считать, что в равенстве (3) $a_{i}$ суть приведенные многочлены, а $\omega_{i}$ - приведенные мономы над $\hat{\mathbf{F}}_{q}$. Поскольку преобразования $\gamma_{i}, i=1, \ldots, k_{0}$, попарно различны, мономы $\omega_{i}$ тоже попарно различны, а тогда из равенства (3) следует, что все $a_{i}, i=1, \ldots, k_{0}$, равны нулю как многочлены и как преобразования. Пришли к противоречию.

Теорема доказана.

В дальнейшем для любой подсистемы $\Gamma$ системы $T$ будем обозначать через $P\left(F_{q}\right)\langle\Gamma\rangle$ подмодуль $P\left(F_{q}\right) P(S)$, порожденный $\Gamma$.

Построенная система образующих модуля $P\left(\mathrm{~F}_{q}\right) P(S)$ позволяет найти его мощность в случае $e \leqslant 1+\delta$. Данному вопросу посвящен следующий параграф.

\section{4. О числе полиномиальных преобразований}

Всюду в этом параграфе предполагается, что $e \leqslant 1+\delta$.

Пусть $x_{i}, i=1, \ldots, \delta,-$ многочлены из $\mathbf{F}_{q}\left[x_{1}, x_{2}, \ldots, x_{\delta}\right]$. Рассмотрим множество векторов над $\mathbf{N}_{0} \cup\left\{x_{1}, x_{2}, \ldots, x_{\delta}\right\}$ вида

$$
\left(j_{0}, x_{i_{1}}, j_{1}, \ldots, x_{i_{k}}, j_{k}\right)
$$

по всем $k \in \mathbf{N}$ с ограничениями $\sum_{l=0}^{k} j_{l} \leqslant e-1$ и $j_{l} \geqslant i_{l}$ для всех $l=1, \ldots, k$. Каждому вектору вида (4) сопоставим преобразование из множества $T$

$$
\hat{\pi}^{j_{0}} \varphi_{i_{1}} \hat{\pi}^{j_{1}} \varphi_{i_{2}} \hat{\pi}^{j_{2}} \ldots \varphi_{i_{k}} \hat{\pi}^{j_{k}}
$$

Будем говорить, что переменная $x_{i}, i \in\{1, \ldots, \delta\}$, имеет вхождение $c \in \mathbf{N}_{0}$ в векторе (4), если существует позиция в векторе (4), на которой стоит $x_{i}$, и сумма чисел, стоящих левее этой позиции, равна $c$. Упорядоченную по возрастанию совокупность вхождений переменной $x_{i}$ будем называть ее списком вхождений. По спискам вхождений $x_{i}$ для всех $i=1, \ldots, \delta$ однозначно восстанавливается вектор (4). Покажем, что преобразование (5) однозначно определяет список вхождений всякой переменной $x_{i}, i=1, \ldots, \delta$.

Поскольку $\hat{\pi} \varphi_{i}=\sigma\left(\varphi_{i}\right) \hat{\pi}$, преобразование (5) можно записать в виде

$$
\sigma^{c_{1}}\left(\varphi_{i_{1}}\right) \sigma^{c_{2}}\left(\varphi_{i_{2}}\right) \ldots \sigma^{c_{k}}\left(\varphi_{i_{k}}\right) \hat{\pi}^{l_{1}+l_{1}+\ldots+l_{k}}
$$

где

$$
c_{i}=\sum_{l<i} j_{l}
$$

Так как $e \leqslant 1+\delta$,

$$
c_{i} \in\{0,1, \ldots, \delta-1\}, \quad i \in\{1, \ldots, k\} .
$$

Пусть числа $d_{i}, i=1, \ldots, \delta-1$, определены, как в параграфе 2 , и $d_{0}=1$. Тогда для любого $c \in\{0,1, \ldots, \delta-1\}$ справедливо равенство $\sigma^{c}\left(\varphi_{i}\right)=\varphi_{i}{ }^{d_{c}}$ и преобразование (5) представляется в виде

$$
\omega\left(\varphi_{1}, \varphi_{2}, \ldots, \varphi_{\delta}\right) \hat{\pi}^{l_{0}+l_{1}+\ldots+l_{k}}
$$

где

$$
\omega=x_{i_{1}}^{d_{c_{1}}} x_{i_{2}}^{d_{c_{2}}} \ldots x_{i_{k}}^{d_{c_{k}}} \in \hat{\mathbf{F}}_{q}\left[x_{1}, x_{2}, \ldots, x_{\delta}\right]
$$


Пусть для всех $i=1, \ldots, \delta$ список вхождений $x_{i}$ есть $c_{1}^{(i)}, c_{2}^{(i)}, \ldots, c_{n_{i}}^{(i)}$. Тогда все $c_{j}^{(i)}$ принадлежат множеству $\left\{c_{1}, c_{2}, \ldots, c_{k}\right\}$ и справедливо равенство

$$
\operatorname{deg}_{x_{i}} \omega=d_{c_{1}^{(i)}}+d_{c_{2}^{(i)}}+\ldots+d_{c_{n_{i}}^{(i)}}
$$

В силу неравенства

$$
d_{c_{1}^{(i)}}+d_{c_{2}^{(i)}}+\ldots+d_{c_{n_{i}}^{(i)}} \leqslant 1+p+p^{2}+\ldots+p^{t-1}<q,
$$

моном $\omega$ является приведенным, а значит, однозначно определяется преобразованием (5).

Для всех $i=1, \ldots, \delta$ числа $c_{j}^{(i)}, j=1, \ldots, n_{i}$, попарно различны и принадлежат множеству $\{0,1, \ldots, \delta-1\}$. Числа $d_{c}, c=0,1, \ldots, \delta-1$, суть степени $p$, причем разным $c$ соответствуют разные $d_{c}$ (см. параграф 2). Следовательно, представляя $\operatorname{deg}_{x_{i}} \omega$ в виде $p$-ичного разложения, по моному $\omega$ можно однозначно определить все $d_{c_{j}^{(i)}}$, а значит, и все $c_{j}^{(i)}$. Таким образом, преобразование (5) однозначно определяет собою список вхождений $x_{i}$ для всех $i=1, \ldots, \delta$ и, следовательно, вектор (4).

Базис $T$ состоит из преобразований $1, \hat{\pi}, \hat{\pi}^{2}, \ldots, \hat{\pi}^{e-1}$ и преобразований вида (5). Поэтому величина $|T|-e$ равна числу векторов вида (4). Если положить $\tau_{l}=j_{l}-i_{l}$, $l \geqslant 1, \tau_{0}=j_{0}$, то множество векторов вида (4) равномощно множеству векторов

$$
\left(\tau_{0}, x_{i_{1}}, \tau_{1}, x_{i_{2}}, \tau_{2}, \ldots, x_{i_{k}}, \tau_{k}\right)
$$

по всем $k \in \mathbf{N}$ с ограничениями

$$
\sum_{l=0}^{k} \tau_{l}+\sum_{l=1}^{k} i_{l} \leqslant e-1, \quad i_{l} \in\{1, \ldots, \delta\}, \quad \tau_{l} \geqslant 0
$$

для всех $l=1, \ldots, k$.

Если

$$
s=\sum_{l=0}^{k} \tau_{l}+\sum_{l=1}^{k} i_{l}, \quad n=i_{1}+i_{2}+\ldots+i_{k},
$$

To

$$
|T|=e+\sum_{s=1}^{e-1} \sum_{n=1}^{s} \sum_{k=1}^{n}\left(\begin{array}{c}
n-1 \\
k-1
\end{array}\right)\left(\begin{array}{c}
s-n+k \\
k
\end{array}\right) .
$$

Действительно, $\left(\begin{array}{c}n-1 \\ k-1\end{array}\right)$ есть число разбиений $n$ на сумму $k$ натуральных слагаемых, а $\left(\begin{array}{c}s-n+k \\ k\end{array}\right)$ есть число разбиений $s-n$ на сумму $k+1$ целых неотрицательных слагаемых.

Комбинируя (7) и теорему 2 , получаем, что справедлива следующая теорема.

Теорема 3. Если $S$ есть $G E O$-кольцо простой характеристики и $е \leqslant 1+\delta$, ранг $\rho$ модуля ${ }_{P\left(\mathbf{F}_{q}\right)} P(S)$ удовлетворяет равенству

$$
\rho=e+\sum_{s=1}^{e-1} \sum_{n=1}^{s} \sum_{k=1}^{n}\left(\begin{array}{c}
n-1 \\
k-1
\end{array}\right)\left(\begin{array}{c}
s-n+k \\
k
\end{array}\right)
$$


Теорема 3 отвечает на вопрос о числе полиномиальных преобразований в случае $e \leqslant 1+\delta$. Полученную величину полезно сравнить с $|P(R)|$, где $R \cong \mathbf{F}_{q}[z] / z^{e}$. Для этого докажем следующее утверждение.

Следствие 2. В условиях теоремы 3 , если $e \leqslant 1+\delta$, то

$$
\rho \geqslant 2^{e+1}-\frac{e(e+1)}{2}-2
$$

Доказательство. Пусть $\tau_{0}$ меняется в пределах от 0 до $s-n$, а остальные $\tau_{i}$ равны 0. Тогда

$$
\rho=|T| \geqslant e+\sum_{s=1}^{e-1} \sum_{n=1}^{s}(s-n+1) \sum_{k=1}^{n}\left(\begin{array}{l}
n-1 \\
k-1
\end{array}\right),
$$

и следовательно, имеет место цепочка соотношений

$$
\begin{aligned}
\rho & \geqslant e+\sum_{s=1}^{e-1} \sum_{n=1}^{s}(s-n+1) 2^{n-1} \\
& =e+\sum_{s=1}^{e-1} s \sum_{n=1}^{s} 2^{n-1}-\sum_{s=1}^{e-1} \sum_{n=1}^{s} n 2^{n-1}+\sum_{s=1}^{e-1} \sum_{n=1}^{s} 2^{n-1} \\
& =e+\sum_{s=1}^{e-1} s\left(2^{s}-1\right)-\sum_{s=1}^{e-1}\left(s 2^{s}-2^{s}+1\right)+\sum_{s=1}^{e-1}\left(2^{s}-1\right) \\
& =e-\sum_{s=1}^{e-1} s+\sum_{s=1}^{e-1} 2^{s}-(e-1)+2\left(2^{e-1}-1\right)-(e-1) \\
& =2^{e+1}-\frac{e(e+1)}{2}-2 .
\end{aligned}
$$

Следствие доказано.

Из результатов работы [4] следует, что

$$
\frac{\log _{q}|P(R)|}{q} \leqslant \frac{e(e+1)}{2} .
$$

Тогда в силу следствия 2 из теоремы 3

$$
\frac{\log _{q}|P(S)|}{q} \geqslant 2^{e+1}-\frac{e(e+1)}{2}-2 \geqslant \frac{e(e+1)}{2} \geqslant \frac{\log _{q}|P(R)|}{q},
$$

причем неравенства одновременно обращаются в равенства, если и только если $e \leqslant 2$. Таким образом, при условии $3 \leqslant e \leqslant 1+\delta$ число полиномиальных преобразований некоммутативного кольца $S$ существенно больше, числа полиномиальных преобразований цепного коммутативного кольца той же мощности и характеристики.

В следующем параграфе будут изучаться полиномиальные подстановки. 


\section{5. Полиномиальные подстановки}

Если рассматривать $S$ как арифметическое векторное пространство над полем $\mathbf{F}_{q}$, то всякое полиномиальное преобразование $f$ кольца $S$ является треугольным. Более того, если $h_{s}\left(x_{0}, x_{1}, \ldots, x_{s}\right)$ есть приведенный многочлен, задающий координатную функщию $f_{s}$, то для всех $s=1, \ldots, e-1$ выполняется равенство $\operatorname{deg}_{x_{s}} h_{s}=1$. Поэтому для всех $s=1, \ldots, e-1$ координатная функция $f_{s}$ однозначно представляется в виде

$$
f_{s}=\alpha_{s}\left(\varphi_{0}, \varphi_{1}, \varphi_{2}, \ldots, \varphi_{s-1}\right)+\psi_{s}\left(\varphi_{0}\right) \varphi_{s},
$$

где $\alpha_{s}, \psi_{s}$ суть приведенные многочлены над полем $\hat{\mathbf{F}}_{q}$. Пусть $T$ - построенная в параграфе 3 система образующих модуля $P\left(\mathrm{~F}_{q}\right) P(S)$ и $\Gamma \subseteq T-$ базис этого модуля. Обозначим

$$
\Gamma_{0}=\Gamma \backslash\left\{\hat{1}, \varepsilon_{1}, \varepsilon_{2}, \ldots, \varepsilon_{\delta}\right\}
$$

Система $\Gamma_{0}$ представляет собой некоторую совокупность преобразований вида

$$
\hat{\pi}^{\tau_{0}} \varepsilon_{i_{1}} \hat{\pi}^{\tau_{1}} \varepsilon_{i_{2}} \hat{\pi}^{\tau_{2}} \ldots \varepsilon_{i_{k}} \hat{\pi}^{\tau_{k}}
$$

где $k \geqslant 0, i_{l} \in\{1, \ldots, \delta\}, \tau_{l} \geqslant 0$, и если $k=0$, то $\tau_{0} \geqslant 1$, а если $k=1$, то $\tau_{0}+\tau_{1} \geqslant 1$. Координатные функции таких преобразований имеют вид

$$
f_{0}=0, \quad f_{s}=\alpha_{s}\left(\varphi_{0}, \varphi_{1}, \varphi_{2}, \ldots, \varphi_{s-1}\right)
$$

для всех $s=1, \ldots, e-1$. Преобразования вида (9) образуют левый $P\left(\mathbf{F}_{q}\right)$-модуль, поэтому все преобразования из модуля $P\left(\mathbf{F}_{q}\right)\left\langle\Gamma_{0}\right\rangle$ имеют вид (9). Данное наблюдение позволяет доказать следующую лемму.

Лемма 4. Справедливо следующее разложение $P\left(\mathbf{F}_{q}\right) P(S)$ в прямую сумму собственных подмодулей:

$$
P\left(\mathbf{F}_{q}\right) P(S)=P\left(\mathbf{F}_{q}\right)\left\langle\Gamma_{0}\right\rangle \dot{+} P\left(\mathbf{F}_{q}\right)\left\langle\hat{1}, \varepsilon_{1}, \varepsilon_{2}, \ldots, \varepsilon_{\delta}\right\rangle .
$$

Доказательство. Поскольку $\Gamma \subseteq \Gamma_{0} \cup\left\{\hat{1}, \varepsilon_{1}, \varepsilon_{2}, \ldots, \varepsilon_{\delta}\right\}$, разложение $P\left(\mathbf{F}_{q}\right) P(S)$ в сумму указанных подмодулей очевидно. Осталось доказать, что сумма является прямой. Пусть 0 представляется в виде $\gamma^{\prime}+\gamma^{\prime \prime}$, где $\gamma^{\prime} \in P\left(\mathbf{F}_{q}\right)\left\langle\Gamma_{0}\right\rangle, \gamma^{\prime \prime} \in P\left(\mathbf{F}_{q}\right)\left\langle\hat{1}, \varepsilon_{1}, \varepsilon_{2}, \ldots, \varepsilon_{\delta}\right\rangle$. По определению преобразований $\varepsilon_{j}, j=1, \ldots, \delta$, и в силу однозначности разложения (8) координатные функции преобразования $\gamma^{\prime \prime}$ имеют вид (9) тогда и только тогда, когда $\gamma^{\prime \prime}=0$. Однако, $\gamma^{\prime \prime}=-\gamma^{\prime} \in P\left(\mathbf{F}_{q}\right)\left\langle\Gamma_{0}\right\rangle$, а значит, координатные функции $\gamma^{\prime \prime}$ имеют вид (9).

Лемма доказана.

Обозначим через $\delta_{0}$ минимум из чисел $e-1$ и $\delta$. По лемме 4

$$
\Gamma=\Gamma_{0} \cup\left\{\hat{1}, \varepsilon_{1}, \varepsilon_{2}, \ldots, \varepsilon_{\delta_{0}}\right\}
$$

и при этом $\Gamma_{0} \cap\left\{\hat{1}, \varepsilon_{1}, \varepsilon_{2}, \ldots, \varepsilon_{\delta_{0}}\right\}=\varnothing$. Тогда для всех $s=1, \ldots, \delta_{0}$ преобразования, задающиеся многочленами $\psi_{s}$, суть коэффициенты при $\varepsilon_{s}$, а $f_{0}$ есть коэффициент при $\hat{1}$ в разложении $f$ по базису $\Gamma$.

В силу вышесказанного для любых $s \geqslant 1, i \geqslant 0$ таких, что выполнено соотношение $s+i \delta \leqslant e-1$, справедливо равенство $\psi_{s+i \delta}=\psi_{s}$. Треугольное преобразование биективно тогда и только тогда, когда каждая из его координатных функций биективна по последней 
переменной. Следовательно, полиномиальное преобразование $f$ биективно тогда и только тогда, когда $f_{0}$ есть биективное преобразование $\mathbf{F}_{q}$ и все $\psi_{s}, s=1, \ldots, \delta_{0}$, суть многочлены, не имеющие корней. Если обозначить $\left|\Gamma_{0}\right|$ через $\rho_{0}$, то число полиномиальных подстановок выражается формулой

$$
|B(S)|=q !(q-1)^{q \delta_{0}} q^{\rho_{0} q} .
$$

Действительно, $q$ ! есть число способов, которыми можно выбрать биективное преобразование $f_{0}$, а $(q-1)^{q \delta_{0}}$ есть число способов, которыми можно выбрать вектор $\left(\psi_{1}, \psi_{2}, \ldots, \psi_{\delta_{0}}\right)$ так, чтобы ни один из многочленов $\psi_{s}$ не имел корней. Если $\rho$ есть ранг модуля $P\left(\mathbf{F}_{q}\right) P(S)$, то $|P(S)|=q^{\rho q}$ и $\rho_{0}=\rho-\delta_{0}-1$. Следовательно, справедлива следующая теорема.

Теорема 4. Если $S-G E O$-кольчо простой характеристики и $\delta_{0}=\min \{e-1, \delta\}$, то число полиномиальных подстановок выражается формулой

$$
|B(S)|=\frac{q !(q-1)^{\delta_{(1)}}}{q^{\left(\delta_{0}+1\right) q}}|P(S)| .
$$

Таким образом, вопрос о числе полиномиальных подстановок сведен к вопросу о числе полиномиальных преобразований. Теорема 4 является обобщением соответствующего результата работы [4] на некоммутативный случай. Заметим также, что биективность полиномиального преобразования $f$ эквивалентна его биективности по модулю $\pi^{1+\delta_{0}}($ в коммутативном случае - по модулю $\pi^{2}$ ).

Назовем преобразование $f \in P(S)$ транзитивным, если его граф представляет собой цикл длины $|S|$.

Теорема 5. Если $S$ - некоммутативное GEO-кольчо простой характеристики, то в кольче $P(S)$ нет транзитивных преобразований.

Доказательство. Проведем доказательство от противного. Пусть $f$ - транзитивное полиномиальное преобразование. Так как $S$ некоммутативно, $e \geqslant 2$. Рассмотрим первые две координатные функции преобразования $f$ :

$$
\begin{aligned}
& f_{0}=\alpha_{0}\left(\varphi_{0}\right), \\
& f_{1}=\alpha_{1}\left(\varphi_{0}\right)+\varphi_{1} \psi_{1}\left(\varphi_{0}\right)
\end{aligned}
$$

Всякому $b \in \hat{\mathbf{F}}_{q}$ сопоставим преобразование $g_{b}$ поля $\hat{\mathbf{F}}_{q}$, определяемое равенством

$$
g_{b}(u)=\alpha_{1}(b)+u \psi_{1}(b)
$$

для всех $u \in \hat{\mathbf{F}}_{q}$.

Поскольку преобразование $f$ транзитивно, оно транзитивно по модулю $\pi$ и по модулю $\pi^{2}$. Это означает, что если последовательность $\left\{b_{i}\right\}_{i=0}^{\infty}$ элементов поля $\hat{\mathbf{F}}_{q}$ получена по правилу $b_{i+1}=\alpha_{0}\left(b_{i}\right)$ и о есть операция композиции, то $\hat{\mathbf{F}}_{q}=\left\{b_{0}, b_{1}, \ldots, b_{q-1}\right\}$ и преобразование поля $\hat{\mathbf{F}}_{q}$

$$
g=g_{b_{q-1}} \circ g_{b_{q-2}} \circ \ldots \circ g_{b_{1}} \circ g_{b_{0}}
$$

является транзитивным. Преобразование $g$ задается некоторым многочленом

$$
\alpha x+\beta \in \hat{\mathbf{F}}_{q}[x] .
$$


Поскольку $g$ транзитивно, $\alpha \notin\{0, \hat{1}\}$ (так как $S$ некоммутативно, характеристика поля $p<q$ ). Пусть $g^{(s)}$ есть $s$-я композиционная степень преобразования $g$, то есть композиция $g \circ g \circ \cdots \circ g\left(s\right.$ раз). Индукщией по $s \geqslant 1$ легко показать, что $g^{(s)}(0)=(\alpha-\hat{1})^{-1}\left(\alpha^{s}-\hat{1}\right) \beta$. Отсюда следует, что $g^{(q-1)}(0)=0$. Следовательно, 0 лежит на цикле преобразования $g$ длины, не большей $q-1$. Пришли к противоречию с транзитивностью $g$.

Теорема доказана.

\section{6. Заключение}

Подведем итоги. В данной работе множество полиномиальных преобразований $P(S)$ GEO-кольца $S$ простой характеристики $p$ рассматривается как левый $P\left(\mathbf{F}_{q}\right)$-модуль, где $P\left(\mathbf{F}_{q}\right)$ есть кольцо преобразований поля $\mathbf{F}_{q}$. Доказано, что модуль $P\left(\mathbf{F}_{q}\right) P(S)$ свободен, и построена его система образующих, которая в случае $e \leqslant 1+\delta$, где $\delta-$ порядок автоморфизма $\sigma$ в группе автоморфизмов поля $\mathbf{F}_{q}$, является базисом. Найдена формула, сводящая вопрос о числе полиномиальных подстановок к вопросу о числе полиномиальных преобразований. При условии $e \leqslant 1+\delta$ вычислен ранг модуля $P\left(\mathrm{~F}_{q}\right) P(S)$, и следовательно, число полиномиальных преобразований. Показано, что в этом случае число полиномиальных преобразований некоммутативного кольца $S$ существенно больше числа полиномиальных преобразований цепного коммутативного кольца той же мощности и характеристики. Кроме того, доказано, что если $S$ некоммутативно, то ни одно из полиномиальных преобразований $S$ не является транзитивным.

Автор выражает признательность А. А. Нечаеву за постановку задачи и существенную помощь при подготовке статьи.

\section{Список литературы}

1. Глухов М. М., Елизаров В. П., Нечаев А. А., Алгебра. Гелиос, Москва, 2003.

2. Нечаев А. А., Конечные кольца главных идеалов. Матем. сб. (1973) 6, 350-366.

3. Dickson L. E., General theory of modular invariants. Trans. Amer. Math. Soc. (1909) 10, №2, 123158.

4. Нечаев А. А., Полиномиальные преобразования конечных коммутативных колец главных идеалов. Матем. заметки (1980) 27, №6, 885-889.

Статья поступила 10.11.2003. 